\title{
Autologous lipoinjection of the patulous Eustachian tube: Harvesting, cellular analysis, clinical application and preliminary outcome
}

\author{
Holger Sudhoff*, Matthias Schürmann and Viktoria Brotzmann \\ Department of Otolaryngology, Head and Neck Surgery, Bielefeld Academic Teaching Hospital, Bielefeld, Germany
}

\begin{abstract}
Objective: Autologous lipoinjection for patulous Eustachian tube dysfunction was investigated. We studied the technique of fat harvesting, fat tissue changes due to processing, clinical application and preliminary outcomes.

Design: Prospective pilot cohort study.

Methods: Transnasal endoscopic injection of autologous lipoinjection into the posterior cushin was performed in 8 patients as a new treatment option for patulous Eustachian tube. All patients were followed up 6 months after treatment. For each intervention, 2-3 ml of injectable soft-tissue bulking agent was used. Fat tissue changes due to processing were studied by confocal laser scanning microscopy.

Results: In 2 patients, more than one procedure was necessary. 6 out of 8 patients were satisfied with the result in the follow up and only 1 patient reported no improvement of symptoms. The procedure was minimally invasive, fast and easy to perform.

Implications: There is no gold standard for the therapy of patulous Eustachian tube. Autologous lipoinjection into the posterior cushin of the Eustachian tube is a new minimally invasive therapeutic approach. Given the emerging discussion regarding the type of surgical treatment of patulous Eustachian tube dysfunction on functional outcomes, this is an area for further research.
\end{abstract}

\section{Introduction}

The Eustachian tube (ET) is generally closed but opens temporarily to meet important physiological functions as ventilation and clearance of the middle ear spaces [1-3]. Clinical symptoms of patulous ET (PET) were initially described by Jago in 1858 and stated by Schwartze in 1864 and recognition PET as a entfernen clinical entity $[4,5]$. The incidence of PET is estimated at about $6 \%$ of the population [6]. Only $10-20 \%$ of the persons concerned seek medical help for their complaints [7]. Patients with a patulous ET often describe similar symptoms to those with a chronic obstructive ET dysfunction: a feeling of pressure in the ears intensifying with changes of the atmospheric pressure and negative Valsalva's maneuver. Additionally, a patulous Eustachian tube can cause symptoms like autophony, breath synchronous tinnitus and tympanic membrane excursions, and pressure sensation in the ear and hearing loss. In combination with "sniffing", it can trigger the development of cholesteatoma [7]. Exercise worsens the complaints and patients often mention an improvement of their complaints in the supine position.

There are obvious deficiencies in diagnosing and a lack of effective therapies for PET dysfunction [8-10]. Valsalva's and Toynbee's maneuver and pneumatic otoscopy are easily conducted but lack reliability and quantifiable results. An objective measurement of tubal function is missing so far. A combination of the different available diagnostic tests is used in clinical practice. Besides physical examination, tympanometry is the most significant tool for diagnosing ET dysfunction. In chronic obstructive ET dysfunction, the curve is usually flattened and/or the compliance negative. Other tests like impedance testing in the pressure chamber require expensive equipment but do not yield any unique information. Additionally, most of these tests require an intact tympanic membrane. Functional MRI of the ET as well as videoendoscopy and sonotubometry has not proven to be suitable for routine clinical practice $[11,12]$. PET is diagnosed only through a well-structured examination including patient history, and physical examination with microscopic observation of movements of the tympanic membrane especially during forced nasal breathing. Tympanometry with reflex-decay should also be performed. The compression of the jugular veins for several minutes might be helpful to certify the suspected diagnosis. Compression is expected to improve the symptoms on the ipsilateral side. Endoscopy of the nasopharynx might reveal a longitudinal concavity in the anterolateral wall of the torus tubarius $[13,14]$.

Efficient therapies for PET have not been described so far.

Correspondence to: Prof. Holger Sudhoff, Department of Otolaryngology, Head and Neck Surgery, Bielefeld Academic Teaching Hospital, Teutoburger Str. 50, 33604 Bielefeld, Germany; Tel: +49 521/581-3301; Fax: +49 521/581-3399; E-mail: holger.sudhoff@rub.de

Key words: patulous Eustachian tube, Eustachian tube dysfunction, Tuba aperta, injectable soft-tissue bulking agent

Received: September 28, 2016; Accepted: October 25, 2016; Published: October 31,2016 
Paracentesis and grommet insertion are the standard therapy of PET dysfunction especially in chronic otitis media with effusion. They might also reduce the symptoms of a PET. However, this therapy is only symptomatic. Another attempt on symptomatic therapy is the cartilage reinforcement of the tympanic membrane. As an office test, a small piece of paper can be placed onto of the tympanic membrane [13-15]. Previously we studied the injection of Vox ${ }^{\oplus}$-implants into the torus tubarius [16]. To extend these promising clinical results, we extended the application to autologous lipoinjection. The technique of fat harvesting, fat tissue changes due to processing, clinical application and preliminary outcomes were key focus.

\section{Materials and methods}

All patients with suspected PET are examined in our specialized Eustachian tube clinic. After an in-depth analysis of patient history including change of symptoms in supine position, under exercise and while compressing the Jugular veins etc.. The physical examination includes a complete ENT-status, otomicroscopy with special attention to movements of the tympanic membrane in normal and in forced breathing. Tympanometry with reflex decay and tubomanometry were also performed. Reflex decay displayed a typical saw tooth pattern in patients with PET. Tubomanometry (TMM) was described by Estève in $2001[17,18]$. It allows an assessment of ET function under defined conditions: Swallowing triggers the release of a defined pressure (e.g. 30,40 and 50 mbar) through a nasal applicator. A pressure probe in the ear canal registers pressure changes transmitted though movements of the tympanic membrane or through a perforation in the tympanic membrane. Consequently, TMM is a semi-objective method to record ET function. Pressure curves of the epipharynx and the ear canal are displayed on the monitor of the TMM device and various measuring values are calculated. The opening latency index ( $R$ value) reflects the latency between pressure application in the epipharynx and registration of a pressure change in the ear canal. This latency quantifies ET function: an immediate opening $(\mathrm{R}<1)$ indicates good ET function, a late opening $(\mathrm{R}>1)$ indicates restricted ET function. No opening $(\mathrm{R}$ negative or not measurable) indicates complete obstruction of the ET. In patients with patulous ET, the opening is always immediate. In some cases of patulous ET, fluctuations of the pressure in the outer ear canal can be observed after pressure application in the nose $[17,18]$.

Patients with typical symptoms, immediate opening in TMM, and saw tooth pattern in reflex decay and visible breath synchronous movement of the tympanic membrane were treated with saline nasal spray and estrogen nasal ointment 3 times a day for 6 weeks. Afterwards, the symptoms and results are reevaluated. If the patient is not satisfied with the outcome of the medical treatment, evtl. ersetzen durch: an aoutologous lipoinjection is discussed. The patients were consented and grommets are offered to patients as an alternative. The minimum age was 18 years, and the patients have to be able to give informed consent to participation. A pretreatment horizontal cone beam tomography in sitting position was done and found to facilitate the diagnosis of PET.

Augmentation of the torus tubarius is performed under general anesthesia. After the harvesting of abdominal fat (Figure 1A) with a special (Figure 1B) designed microfat transfer set (Spiggle\&Theis, Overath, Germany). The harvesting needle has lateral positioned opening to suction small pieces of abdominal fat. This is performed under the low vacuum of a $10 \mathrm{ml}$ syringe. After having harvested 2 times $5 \mathrm{ml}$, the syringes are placed into a centrifuge at $3.000 \mathrm{rpms}$ for 3 minutes (Figure 2A). The supernatant was removed and the fat
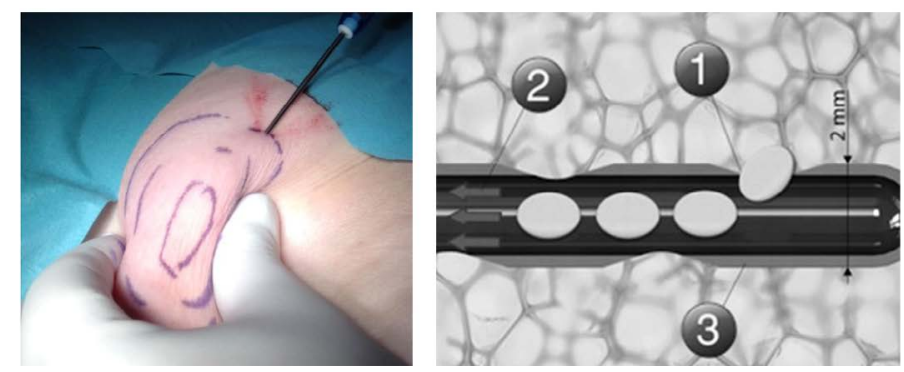

Figure $1 \mathrm{~A}$ and B. Harvesting abdominal fat (A). The harvesting needle allows to obtain a homogenic adipose tissue under a low vacuum (1) Gauged orifices $(600 \mu \mathrm{m})$, (2) sampler, $2 \mathrm{~mm}$ diameter, (3) suction under low vacuum preserving the integrity of fat units (B).

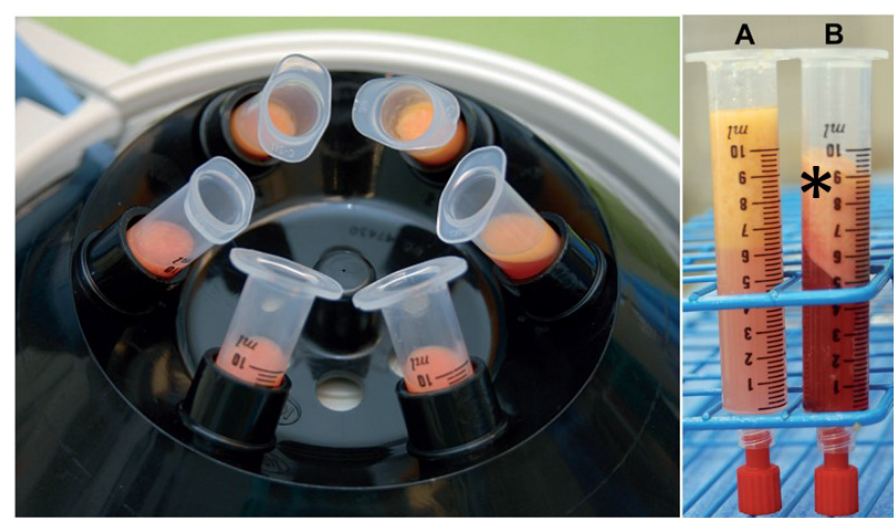

Figure 2. Processing of the harvested fat tissue by centrifugation at $3.000 \mathrm{rpms}$ for $3 \mathrm{~min}$ The pellet will be reinjected after removal of the oily supernatant $\left(^{*}\right)$.

portion collected and a special needle was attached to the syringe under a low vacuum (Figure 2 BA) (Spiggle\&Theis, Overath, Germany). The applicator (Figure $3 \mathrm{~A}$ ) is transorally positioned in front of the torus tubarius under endoscopic control. For visualization we recommend a $70^{\circ}$ Hopkins endoscope in the contralateral nostril. Fat augmentation is injected in two portions submucosal posterior and superior-anterior in the torus tubarius (Figure 3B). The procedure took about 20-30 minutes bilaterally. Patients were discharged from the hospital on postoperative day one. All included patients were reevaluated 6 months postoperatively. A revision with a second injection of autologous lipoinjection is offered in the event of no or little improvement six weeks post-treatment.

\section{Nile red staining and laser scanning microscopy (LSM)}

The fat-tissue samples were transferred to the laboratory on ice directly after the surgical procedure. Fat was harvested before and after centrifugation to investigate the integrity of the fat units. Subsequently the tissue samples were fixed with $4 \%$ paraformaldehyde in phosphate buffered saline (PBS) for $20 \mathrm{~min}$ at room temperature followed by washing in PBS. Afterwards the fat-tissue fragments were transferred into a solution of PBS supplemented with $1 \mu \mathrm{g} / \mathrm{ml} 4^{\prime}, 6$-Diamidin-2phenylindol and $10 \mu \mathrm{g} / \mathrm{ml}$ Nile red and incubated for 15 min under gentle shaking. This staining procedure was followed by two washing steps of $5 \mathrm{~min}$ each in PBS. Finally, the samples were mounted in mowiol and dried overnight. The different fat-tissue samples were characterized by a Zeiss LSM 780 confocal laser scanning microscopy and processed with ImageJ (https://imagej.nih.gov/ij/).

\section{Results}

Between October 2015 and October 2016, 8 patients with patulous 

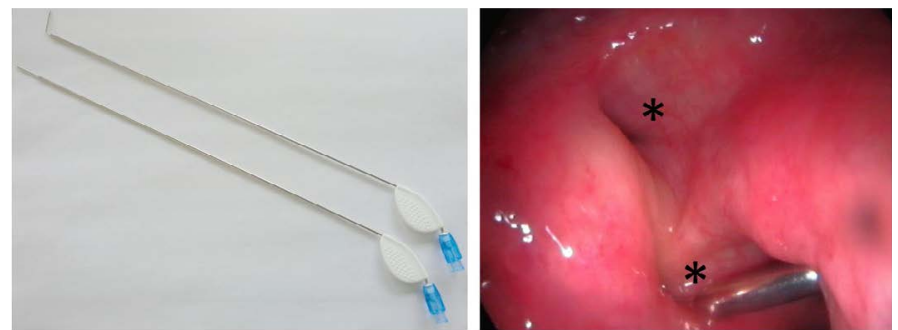

Figure 3. Angulated injection needles designed for the transoral augmentation of the PET Endoscopic view of autologous lipoinjection into the torus tubarius $\left(^{*}\right), 2 \mathrm{ml}$ are generally sufficient for one procedure.

ET were treated with the injection of autologous fat into the PET as described. The mean age of the patients was 39 years, $50 \%$ were female and $50 \%$ male. The major complaints were autophony, pressure in the ear and breath synchronous tympanic tinnitus. Two patients with visible breath synchronous movements of the tympanic membrane. Preoperative cone beam scans of the temporal bone were performed in all patients. Reflex decay testing displayed a typical saw tooth pattern supporting the diagnosis of PET. All 8 patients had no benefit from medical. 5 patients had a history of grommet insertion without success. All patients declined the insertion of grommets. 6 patients had normal hearing, 1 patient a mild sensorineural hearing loss and 1 patient a mild conductive hearing loss. All patients with intact tympanic membranes had a type A tympanogram. TMM revealed an immediate opening of the ET at $30-40$ mbar in 8 out of the 8 patients.

A total of 8 patients suffering from PET were treated: 4 patients on the right, 2 patients on the left side and 2 with bilateral PET. A total of 10 interventions with injection of injection of autologous fat in the torus tubarius were performed. 7 out of 8 patients were treated with a single injection. In 1 patient, repeated injections were necessary. Until today, no serious complications were observed apart from occasional mild epistaxis. Signs of obstruction of the ET or granuloma formation were not observed in our patient collective.

After the first injection of the injection of autologous fat, 7 patients having a significant improvement and 1 patient, no improvement. 7 patients were satisfied with the result after treatment and needed no further interventions. In 1 patient, a second injection of autologous fat injection was performed at least 6 weeks after the first intervention. After the second injection the patient reported a significant improvement.

In summary, 7 out of the 8 patients with complete follow-up were satisfied with the outcome and 1 patient reported improvement after the reinjection. The procedure was fast, easy to perform and minimally invasive with low morbidity. No abdominal hematomas were observed. All 8 patients returned for a complete re-evaluation after the treatment with injection to our clinic. Audiometry, tympanometry and TMM were not altered after treatment. The reflex decay tympanogram revealed a clear reduction or absence of the saw tooth pattern in 7 patients. A second treatment reduced the saw tooth pattern and subjective complaints in the patient that did not show an initial improvement.

Confocal laser scanning microscopic found the single densely packed adipocytes exhibiting a diameter between approximately $70 \mu \mathrm{m}$ and $200 \mu \mathrm{m}$ before and after the centrifugation. The rim of the extracted fat-tissue fragment exhibits some lipid droplets arising from ruptured adipocytes (arrows) after tissue processing (Figure 4A). Beside these adipocytes many cells of the stromal vascular fraction remain intact in both tissues (Figure 4B).
Fluorescence microscopic image of the extracted fat-tissue showed that the majority of tissue is occupied by mature adipocytes tightly packed together in the processed fat tissue (Figure 5). Lipid droplet inside the monovacuolar cells shoved the nucleus to the periphery of the adipocytes pre- and post-processing (arrow). Between the adipocytes the stromal vascular fraction of cells from the fat-tissue remained residing (arrowhead). 3-D images from confocal laser scanning microscopic data showed that the processed fat-tissue fragment had a width of up to a few $\mathrm{mm}$, while its thickness was found to be between 100 and $200 \mu \mathrm{m}$ (Figure 6). Hence its morphology represents a tissue sheet with only a thickness of around 2 mature adipocytes due to processing.

\section{Discussion}

A recent review on the surgical management of PET by Hussein and co-workers included techniques of ET plugging, ventilation tube placement and suture ligation [19]. Less commonly conducted techniques include hydroxyapatite or soft-tissue bulking agent injection, implantation of autologous cartilage, diathermy, gelatin injection, cauterization, pterygoid hamulotomy, and transection/transposition

A

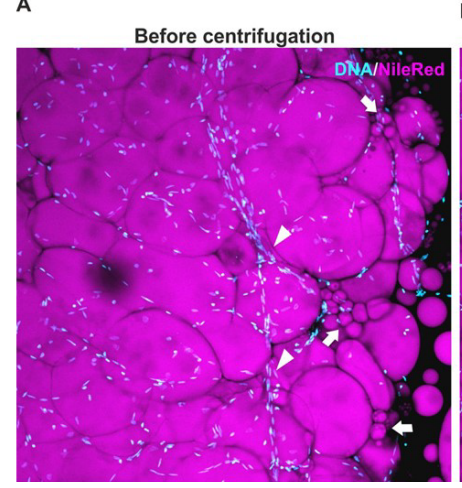

B After centrifugation

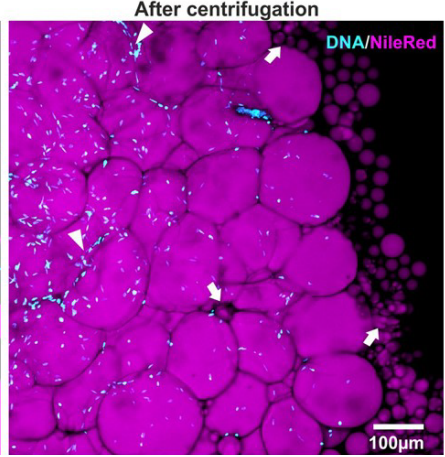

Figure 4. The structure of extracted fat tissue after the centrifugation step: A confocal lase scanning microscopic image of fat-tissue before and after the centrifugation step. The single densely packed adipocytes are visible and exhibiting a diameter between approximately 70 $\mu \mathrm{m}$ and $200 \mu \mathrm{m}$. The rim of the extracted fat-tissue fragment exhibits some lipid droplets arising from ruptured adipocytes (arrows). Beside these adipocytes many cells of the stromal vascular fraction are present in both types of tissue. Notably intact capillaries are present in both type of tissue sample (arrowhead).

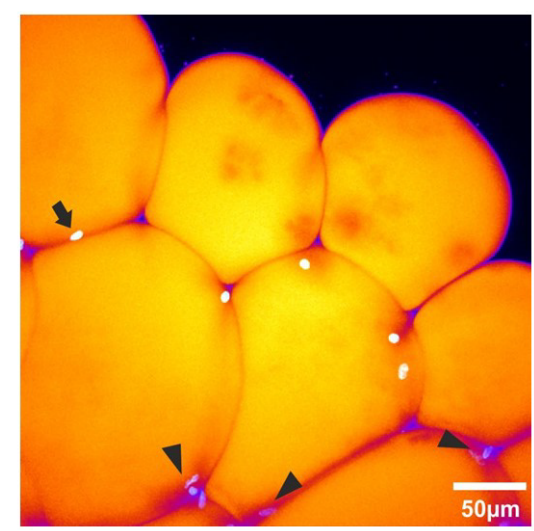

Figure 5. Cellular composition of the extracted fat tissue: Fluorescence microscopic image of the extracted fat-tissue stained with nile red and DAPI. The majority of the tissue is occupied by mature adipocytes tightly packed together. Huge lipid droplet found inside the monovacuolar cells which displaces the nucleus to the periphery of the adipocytes (arrows). In between the adipocytes the stromal vascular fraction of cells from the fat-tissue is residing (arrows). 


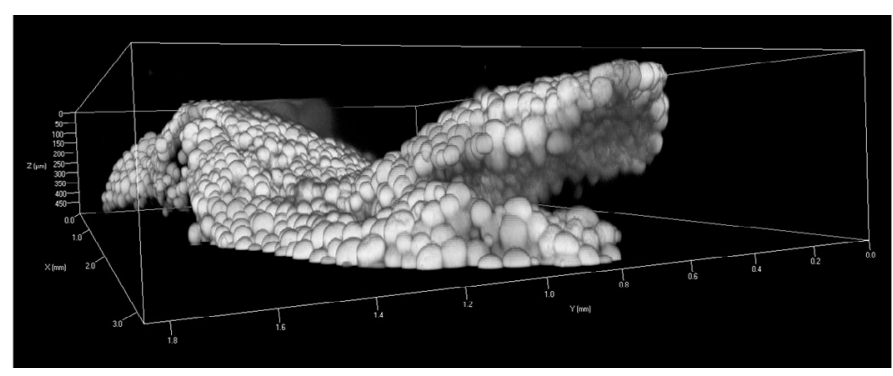

Figure 6. Sheet-like morphology of an extracted fragment after liposuction and centrifugation of fat-tissue: 3 -D reconstruction of a fat tissue fragment from confocal laser scanning microscopic data. The fat-tissue fragment show a width of up to a few $\mathrm{mm}$, while its thickness is only between 100 and $200 \mu \mathrm{m}$. Hence its morphology represents a tissue sheet with only a thickness of around 2 mature adipocytes.

of the tensor veli palatini muscle [16,19-24]. If conservative treatment options do not lead to an improvement of the clinical symptoms, different treatment options for PET are available. Myringotomy and grommet insertion have been demonstrated effective in reducing the symptoms but are no causal therapy [25]. In a retrospective analysis of 46 patients with patulous ET, grommets significantly reduced the symptoms in $53 \%$ of the ears [26]. Another option for a symptomatic treatment is the augmentation of the tympanic membrane with cartilage especially in very atrophic eardrums. To test the effect of the augmentation preoperatively, a small piece of paper can be placed on the eardrum in the office. Surgical options include the injection of different substances such as teflon, gelfoam or paraffin into the pharyngeal ostium of the ET. These treatment options bear significant risks: The injected material might dislocate and cause pharyngeal granuloma [27]. There are also reported cases of cerebral thrombosis due to accidental injection into the internal carotid artery [27]. The injection of autologous fat has also been described as safe and easy to perform. However, significant resorption of this material might compromise the long-term effect [28]. Using our novel technique, we hope to solve this problem as the initial histological results only show minor damage to the harvested tissue due to processing. We assume that smaller fat units will result in a better survival of the autologous fat transplant and a reduced resorption. Recently it has been shown that cell-assisted lipotransfer has better efficacy than conventional fat grafting (non-cellassisted lipotransfer). Nevertheless, this is more applicable to face cases than to breast cases and has not been demonstrated for the Eustachian tube [29]. However, more evidence is needed to prove its survival and long-term clinical effectiveness in larger patient cohorts.

Kobayashi described the Patulous Eustachian tube Plug (PEP) as a promising therapy for a patulous Eustachian tube. The "Kobayashi Plug" made of silicone is inserted into the bony part of the ET via paracentesis. The plug has a length of 23-25 $\mathrm{mm}$. The plug blocks the ET, but grommets are needed to prevent middle ear effusion. According to Kobayashi and co-workers the treatment is effective in about $71 \%$ $[30,31]$. Long-term data to prove the tolerability and possible side effects of this implant are needed. It is important to emphasize that a grommet is necessary as long as the plug is in position.

The injection of autologous processed abdominal fat in the torus tubarius is a new alternative in the treatment of PET dysfunction. It is easy and efficient to perform. Severe complications or side effects have not been observed so far. The advantage of this treatment is that it is minimally invasive as compared to other methods. All patients were discharged from hospital the morning after the intervention and did not feel adversely affected. Patients have to be informed that repeated procedures may become necessary to resolve all clinical symptoms. We normally try to avoid injecting more than $2 \mathrm{ml}$ in one intervention due to the risk of obstruction of the ET. weg Since this is a novel treatment option, only 8 patients were treated so far and long-term follow-up data are not yet available. In the absence of causal therapy for PET dysfunction, further investigations are desirable.

\section{Implications}

The treatment goal for PET is to attain a long-lasting normal ET function. The autologous lipoinjection technique provides chronic PET dysfunction patients with a less invasive treatment option. Further, a method to evaluate the severity of PET such as through a grading system has yet to be developed and standardized. The integrity and long-term results of the injected fat needs to be followed by larger prospective clinical studies.

\section{References}

1. Yamaguchi N, Sando I, Hashida Y, Takahashi H, Matsune S (1990) Histologic study of eustachian tube cartilage with and without congenital anomalies: A preliminary study. Ann Otol Rhinol Laryngol 99: 984-987. [Crossref]

2. Virtanen H (1978) Sonotubometry. An acoustical method for objective measurement of auditory tubal opening. Acta Otolaryngol 86: 93-103. [Crossref]

3. Cantekin EI, Saez CA, Bluestone CD, Bern SA (1979) Airflow through the eustachian tube. Ann Otol Rhinol Laryngol 88: 603-612. [Crossref]

4. Jago J (1858) On the functions of the tympanum. Proc R Soc Lond B Biol Sci 9:134140

5. Schwartze H (1864) Respiratorische Bewegung des Trommelfelles. Arch Ohrenheilk 1:139-140.

6. Reiss M, Reiss G (2000) [Patulous eustachian tube--diagnosis and therapy]. Wien Med Wochenschr 150: 454-456. [Crossref]

7. Henry DF, DiBartolomeo JR (1993) Patulous eustachian tube identification using tympanometry. J Am Acad Audiol 4: 53-57. [Crossref]

8. Poe DS (2007) Diagnosis and management of the patulous eustachian tube. Otol Neurotol 28: 668-677. [Crossref]

9. Pau HW (2011) [Eustachian tube and middle ear mechanics]. HNO 59: 953-963. [Crossref]

10. Di Martino EF (2013) [Eustachian tube function tests: an update]. HNO 61: 467-476. [Crossref]

11. Reiss M, Reiss G (2000) [Patulous eustachian tube--diagnosis and therapy]. Wien Med Wochenschr 150: 454-456. [Crossref]

12. Finkelstein Y, Talmi YP, Rubel Y, Zohar Y (1988) An objective method for evaluation of the patulous eustachian tube by using the middle ear analyzer. Arch Otolaryngol Head Neck Surg 114: 1134-1138. [Crossref]

13. Poe DS (2007) Diagnosis and management of the patulous eustachian tube. Otol Neurotol 28: 668-677. [Crossref]

14. Pau HW (2011) [Eustachian tube and middle ear mechanics]. HNO 59: 953-963. [Crossref]

15. Sudhoff H (2016) Eustachian tube dysfunction. In: Hearing: An Introduction \& Practical Guide, James Tysome, Rahul Kanegaonkar (Editors). CRC Press, Tylor Francis Group 69-71.

16. Schröder S, Lehmann M, Sudhoff HH, Ebmeyer J (2015) Treatment of the patulous Eustachian tube with soft-tissue bulking agent injections. Otol Neurotol 36: 448-452. [Crossref]

17. Esteve D, Dubreuil C, Della Vedova C, et al. (2001) Evaluation par tubomanométrie de la fonction d'ouverture tubaire et de la réponse tympanique chez le sujet normal et chez le sujet porteur d'une otite séro-muqueuse chronique: comparaison des résultats. J Fr ORL 50: 223-231

18. Esteve D (2003) Tubomanometry and pathology. In: B A, ed. Fibrocartilaginous eustachian tube. Middle ear cleft. The Hague: Kugler Publications 159-175.

19. Hussein AA, Adams AS, Turner JH (2015) Surgical management of Patulous Eustachian tube: A systematic review. Laryngoscope 125: 2193-2198. [Crossref] 
20. Vaezeafshar R, Turner JH, Li G, Hwang PH (2014) Endoscopic hydroxyapatite augmentation for patulous Eustachian tube. Laryngoscope 124: 62-66. [Crossref]

21. Robinson PJ, Hazell JW (1989) Patulous eustachian tube syndrome: the relationship with sensorineural hearing loss. Treatment by eustachian tube diathermy. $J$ Laryngol Otol 103: 739-742. [Crossref]

22. Ogawa S, Satoh I, Tanaka H (1976) Patulous Eustachian tube. A new treatment with infusion of absorbable gelatin sponge solution. Arch Otolaryngol 102: 276-280. [Crossref]

23. Virtanen H, Palva T (1982) Surgical treatment of patulous eustachian tube. Arch Otolaryngol 108: 735-739. [Crossref]

24. Stroud MH, Spector GJ, Maisel RH (1974) Patulous eustachian tube syndrome. Preliminary report of the use of the tensor veli palatini transposition procedure. Arch Otolaryngol 99: 419-421. [Crossref]

25. Ikeda R, Oshima T, Oshima H, Miyazaki M, Kikuchi T, et al. (2011) Management of patulous eustachian tube with habitual sniffing. Otol Neurotol 32: 790-793. [Crossref]
26. Chen DA, Luxford WM (1990) Myringotomy and tube for relief of patulous eustachian tube symptoms. Am J Otol 11: 272-273. [Crossref]

27. Kirsch CF, Suh JD, Lufkin RB, Canalis RF (2007) False-positive positron-emission tomography-CT of a Teflon granuloma in the parapharyngeal space occurring after treatment for a patulous eustachian tube. AJNR Am J Neuroradiol 28:1371-1372. [Crossref]

28. Doherty JK, Slattery WH 3rd (2003) Autologous fat grafting for the refractory patulous eustachian tube. Otolaryngol Head Neck Surg 128: 88-91. [Crossref]

29. Zhou Y, Wang J, Li H, Liang X, Bae J, et al. (2016) Efficacy and Safety of Cell-Assisted Lipotransfer: A Systematic Review and Meta-Analysis. Plast Reconstr Surg 137 44e-57e. [Crossref]

30. Ikeda R, Oshima T, Oshima H, Miyazaki M, Kikuchi T, et al. (2011) Management of patulous eustachian tube with habitual sniffing. Otol Neurotol 32: 790-793. [Crossref]

31. Sato T, Kawase T, Yano H, Suetake M, Kobayashi T (2005) Trans-tympanic silicone plug insertion for chronic patulous Eustachian tube. Acta Otolaryngol 125: 1158-1163. [Crossref]

Copyright: (C2016 Sudhoff H. This is an open-access article distributed under the terms of the Creative Commons Attribution License, which permits unrestricted use, distribution, and reproduction in any medium, provided the original author and source are credited. 